\title{
Combining models of behavior with operational data to provide enhanced condition monitoring of AGR cores
}

\author{
Graeme M. West, Christopher J. Wallace and Stephen D.J. McArthur \\ Electronic \& Electrical Engineering, University of Strathclyde, Glasgow, UK
}

\begin{abstract}
Installation of new monitoring equipment in Nuclear Power Plants (NPPs) is often difficult and expensive and therefore maximising the information that can be extracted from existing monitoring equipment is highly desirable. This paper describes the process of combining models derived from laboratory experimentation with current operational plant data to infer an underlying measure of health. A demonstration of this process is provided where the fuel channel bore profile, a measure of core health, is inferred from data gathered during the refueling process of an Advanced Gas-cooled Reactor (AGR) nuclear power plant core. Laboratory simulation was used to generate a model of an interaction between the fuel assembly and the core. This model is used to isolate a single frictional component from a noisy input signal and use this friction component as a measure of health to assess the current condition of the graphite bricks that comprise the core. In addition, the model is used to generate an expected refueling response (the noisy input signal) for a given set of channel bore diameter measurements for either insertion of new fuel or removal of spent fuel, providing validation of the model. This benefit of this work is that it provides a greater understanding of the health of the graphite core, which is important for continued and extended operation of the AGR plants in the UK.
\end{abstract}

Index Terms - Intelligent Systems, Fission Reactor Monitoring, Graphite Ageing

\section{INTRODUCTION}

$\mathrm{T}$ HROUGHOUT the worldwide nuclear industry there is a desire to extend the operational lifetimes of the power plants. For example, in the US the Light Water Reactor Sustainability (LWRS) programme aims to extend the operational lives of the existing plant beyond 60 years (Coble et al., 2012). Similarly, in the UK, the lifetimes of the some of the Advanced Gas-cooled Reactor (AGR) plants have been extended 10 years beyond their original 35 years design life. A significant input into the justification for extended operation is routine assessment of the health of the assets. Broadly, this is undertaken in two ways, firstly through direct inspection of the asset during statutory outages, and secondly through analysis of condition monitoring data gathered during operation. Inspection can provide very detailed and direct information relating to the asset, but at significant cost as the power plant needs to be shut down and is thus not generating electricity. Conversely, online condition monitoring data often typically provides an indirect measure of health (for example, the health of a turbine blade can be inferred from measures of vibration seen on the bearings (Randall, 2004)), but does so in a much more timely manner (many condition monitoring systems gather data continuously).

Data capture and measurement systems in nuclear power plants, however, were rarely designed to measure the degradation of an asset, instead being used to provide information for control and protection purposes. While modern data capture devices and cost of storage of large volumes of data is relatively cheap, there are several barriers to installing new sensing equipment at existing operational stations. Firstly, ensuring that the equipment meets the much higher design and safety requirements of a nuclear installation than typical commercial use. Secondly, physical access to the hostile environment of a nuclear reactor add significant challenges. Thirdly, ensuring that the installation of new equipment does not impact on existing plant equipment and systems that have been approved and licensed. Condition monitoring systems in ageing plant, therefore, tend to be a combination of original sensing equipment with upgraded data capture and storage. Use of existing sensing equipment has its own challenges, such as limited resolution, lack of direct measurement of the degradation parameter and limited expertise within the organisation in the interpretation of the monitoring signals. However, addressing these issues and re-using existing data sources is often much more economically viable than installation of new sensing systems.

This paper explores these challenges through a case study of extracting a health metric of an AGR core from routinely gathered Fuel Grab Load Trace (FGLT) data. The FGLT data is gathered to inform the reactor protection trip system in the case of snagging or dropping of fuel during routine refuelling operation (an advantage of AGR power plant design is that they can be 
refuelled while still generating). It has been established in previous work (West et al., 2006) that FGLT data can also provide useful information relating to variations in the fuel channel bore, an indicator of the health of the graphite core of an AGR. However, this is an indirect measure of core health and in order to maximise the useful information the influence of the parameter associated with core condition (the frictional response of a set of stabilising brushes which contact the fuel channel bore) needs to be isolated from the other sources of information which contribute to the overall FGLT, such as other friction effects as well as aerodynamic effects caused by the core coolant. Direct measurement of these components is not possible, instead representative models are used to simulate the response. These models are validated through laboratory based experimental rig work (Skelton, 2007).

The first section of this paper provides a description of general approach adopted, while the second section provides background information relating to the specifics of the AGR refuelling process. The next sections detail the modelling of the three main components of the FGLT; lower brush friction, upper brush friction and aerodynamic effects. There then follows a description of how bore measurements taken during inspection are used to generate an expected FGLT response for a given set of core conditions, providing confidence that the model is correct. The model is then fed with unseen FGLT data to estimate the equivalent bore measurements, which are in turn compared to measured values of channel bore taken from an associated inspection campaign.

\section{APPROACH}

The approach adopted here is to apply knowledge and a model to existing condition monitoring data in order to decompose a measured signal into its component parts, and therefore estimate an un-measured property. There are three stages to this approach, creation of the model, validation of the model and use of the model to estimate the un-measured property. When creating the model, it is important that the contribution of components which comprise the final signal are understood, even if they are not directly measured. This is as opposed to blind source separation techniques, which take multiple measurements of an event and apply mathematical procedures to isolate the sub-components of the signal. Each component of the model should be tested in isolation where possible. As raw input data for the components in isolation is not directly available, it is necessary to generate this data, either from laboratory experimentation or through direct measurement of the component during operational outages. In order to validate the model, a set of known input values for the un-measured parameters are applied to generate simulated data output which is then compared to a suitable set of output measurements. The model may be refined if required, but once the two results are suitably similar then the model can be used. Fig.1. illustrates this approach with the top half representing the model validation and the bottom half representing the model use. Note the difference in direction of the flow of data through the model is reversed for the use of the model.

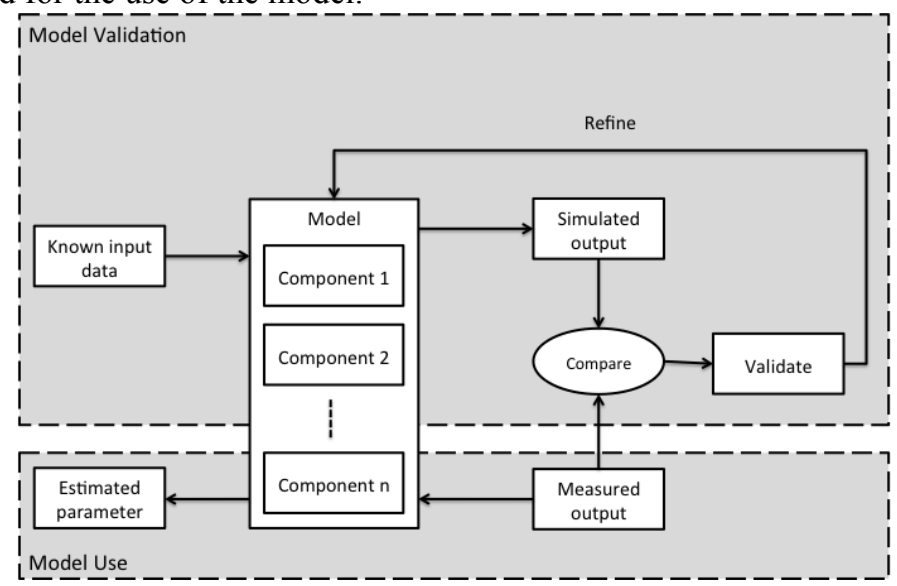

Fig.1. Diagram illustrating the validation and use of the model. The top half of the diagram shows the model inputted with raw data for the interest and a simulated response being generated. This is compared to the actual response and any discrepancies used to refine the model. The lower half of the diagram illustrates the use of the measured data to generate the estimated parameter, using the same model.

\section{ADVANCED GAS-COOLED REACTORS}

\section{Overview}

The AGR design of nuclear power plant is the predominant design currently in operation within the UK, accounting for 7 out of the 9 currently operating plants. The core of an AGR reactor is constructed from thousands of hollow, cylindrical graphite bricks that lock together to form a lattice. The graphite bricks form the physical structure of the core to house the fuel and to allow passage of coolant gas around the core and in addition, the graphite also acts as the moderator (Steer, 2005). Inspection of the core is undertaken during planned outages every 2-3 years where the inner bore diameter of these hollow cylindrical graphite bricks is measured (Cole-Baker and Reed, 2007). Undertaking these inspections is costly as the station needs to be shut down and the channel emptied of fuel, before the inspection unit can be inserted. As a result, only a small percentage of the fuel 
channels, typically 31 out of the 308 for the oldest reactors are inspected every three years. In addition to the inspection regime, data is also gathered during routine plant operation which provides information relating to the health of the core. When refuelling of the reactor occurs, typically every 6-8 weeks, the apparent weight of the fuel assembly is measured, with factors such as friction based on the bore diameter influencing the measurement. This measurement of apparent load is termed the fuel grab load trace (FGLT). Isolating the contribution of the bore variation to the overall FGLT can provide a better understanding of the health of the graphite, and can improve the identification of anomalies within the core.

\section{Ageing of graphite}

The major life-limiting factor of the AGR plants is the condition of the graphite bricks that comprise the core of the nuclear reactors (Shennan, 1983). At very high neutron doses such as those found in the AGR cores, the graphite deforms as a result of the effects of stress and irradiation and is termed radiation creep (Simmons and Perks, 1965) in addition to the graphite dimensional change with irradiation detailed by (Brocklehurst and Kelly, 1993, Neighbour, 2000, Marsden et al, 2005 and Marsden et al, 2008). One consequence of these dimensional changes is that the internal bore diameter of the fuel channels will change. If the distortion is severe enough, then this may start to impact on the movement of fuel, which has major implications for continued operation of the station.

\section{Related work}

Automated analysis of FGLT data has become possible through the development of the BETA system for identifying anomalous behavior within FGLT using various intelligent systems techniques (West et al., 2006, Stephen et al. 2009 and West et al., 2011). The approach adopted in this system is to compare new refueling events with an empirically defined benchmark. This benchmark is based on the full FGLT rather than on the contribution made by the individual frictional component (and thus bore diameter) of the lower stabilizing brush.

Initial work reported in Yang et al, 2010 attempts to isolate the friction and aerodynamic effects, however, the separation of the effect of the upper and lower brushes was not satisfactorily achieved, instead preferring a lumped friction model. In addition, though the separation of the aerodynamic forces from the friction was demonstrated, no general model was established.

In He and Gotts, 2005 a computational study of the effects of graphite brick distortion is carried out using finite element analysis, though the goal was to determine what the results of distortion would have on temperature within the core. Although not directly applicable here, similar challenges relating to the validation of results were addressed.

The issue of isolating channel bore measurements from refueling data is particular to the AGR design, however, the wider issues of analyzing condition monitoring data to provide support to continued and extended operation of nuclear power plants is important to all designs of reactor. In ageing nuclear plant, the role of online condition monitoring is become more prevalent, as discussed in Hines and Seibert, 2006, Hashemian, 2010, Hashemian 2011 and Ma and Jiang, 2011.

\section{MODELING}

It is not practical to obtain isolated measured data for each of the components involved in the refueling process. For example it is not practical to remove the upper set of stabilizing brushes and insert a fuel stringer with only lower stabilizing brushes into fuel channel. Therefore, modeling the process provides the best alternative for approximating the response of the individual components.

\section{Lower brush friction}

A model for the lower brush friction has been developed based on theoretical understanding of the interaction between the brushes and the graphite brick and will now be described. This model is then compared to results from experimental data to provide confidence in the accuracy of the model.

\section{Experimental data}

A series of experiments was undertaken by AMEC NNC Limited (an engineering consultancy company which is now part of the AMEC plc) in 2006 to determine the effect of fuel channel bore shrinkage on brush friction and to determine whether a narrow bore would contribute enough friction force to increase the apparent weight of the fuel assembly beyond the safety limits and cause the fuel machine to trip the reactor (Skelton, 2007). One experiment involved machining the bore of a surplus fuel brick from $263 \mathrm{~mm}$ to $251 \mathrm{~mm}$ in steps of $2 \mathrm{~mm}$ to investigate the friction contribution at various bore diameters. A drawing of the brick is provided in Fig.2. Real stabilizing brushes were fitted to a "dummy" fuel assembly nose and the completed assembly pulled through the machined brick while measuring the resulting load caused by the brush friction. A drawing of a typical fuel assembly nose arrangement, along with a drawing and photograph of a cross-section of the lower stabilizing brushes is shown in Fig.3. 

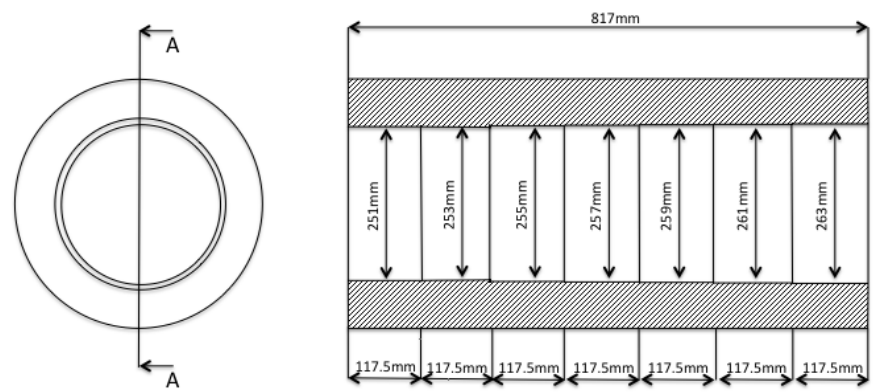

Fig.2. Drawing showing the test brick with inner bore machine from $251 \mathrm{~mm}$ to $263 \mathrm{~mm}$.

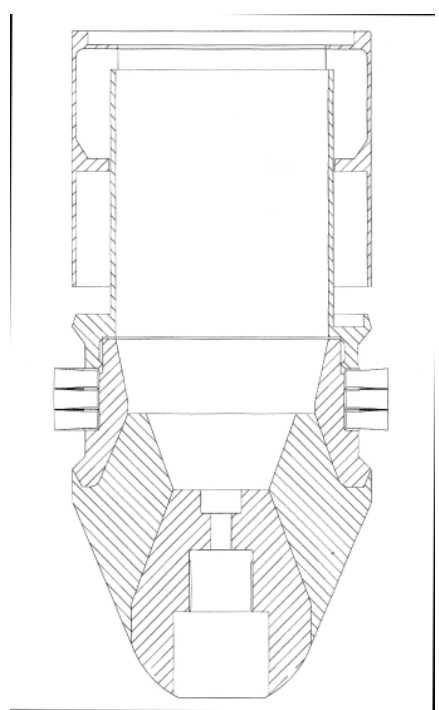

(a)

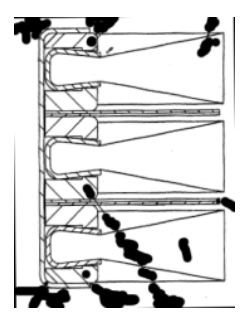

(b)

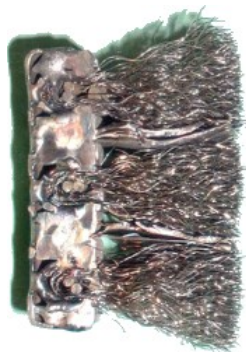

(c)

Fig.3. (a) Diagram of the nose assembly, (b) diagram of a cross-section of the lower stabilizing brush and (c) a photo of a cross-section of the lower stabilizing brush

The measured response as the fuel assembly nose is pulled through the test brick for a single run is shown in Fig.4. starting from a bore of $263 \mathrm{~mm}$ and traversing through the progressively narrower bores. From the plot the frictional load increases as the bore narrows, and does so in a non-linear manner. This is consistent with the idea that the brushes being wider than bore are pushing back on the brick bore, creating the normal force $(\mathrm{N})$ which is proportional to the frictional force (f) by the coefficient of friction $(\mu)$, and described by:

$$
\mu=\frac{f}{N}
$$

At the transition points between the different bores, a set of three spikes is present. The spikes are due to each of the sets of brushes passing the bore transition point. This phenomena is similar to that of static friction, where an increased force is required to initiate movement.

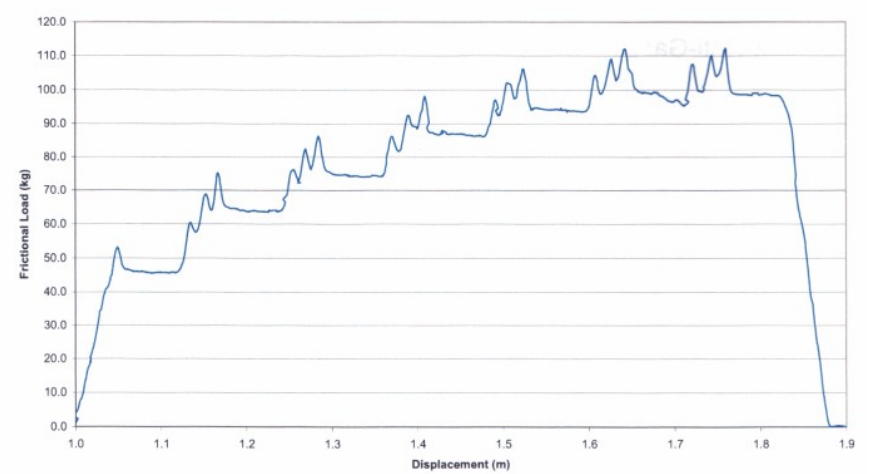

Fig.4. Drawing showing the test brick with inner bore machine from $251 \mathrm{~mm}$ to $263 \mathrm{~mm}$ 
Model of lower brush

A model of the lower brush friction load was created which takes an array of bore diameters as an input and generates the frictional load response. The output is the summation of the kinetic and static responses of each individual brush.

Through linear regression, the relationship between bore $(\phi)$ and kinetic frictional response for three combined brushes $(F r)$ was approximated to be:

$$
F r=-0.0249846 \phi^{3}+18.8256 \phi^{2}-4729.3581 \phi+396226
$$

Due to physical limitations of the bricks, valid values of bore range from about $251 \mathrm{~mm}$ to $263 \mathrm{~mm}$, and this is shown in Fig.5. and the equations is only valid over these ranges.

\section{Brush Friction Characteristic}

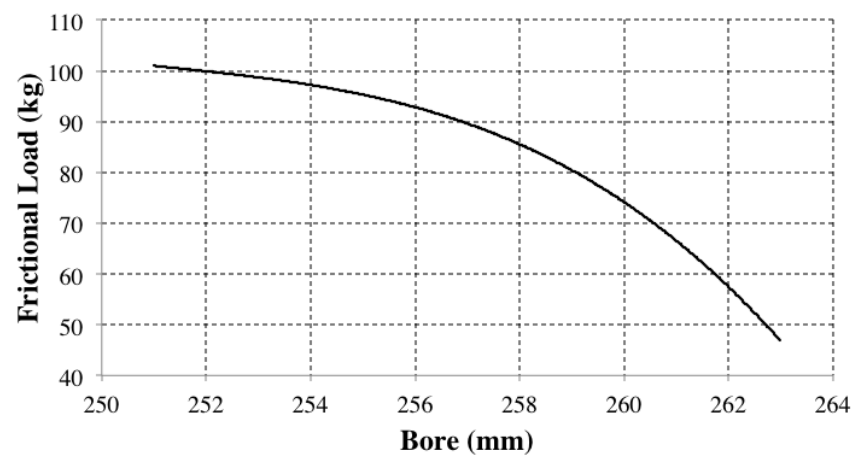

Fig.5. Plot of variation of brush friction load against bore obtained from experimentation

For the static frictional response of the individual brushes, the approach to generate an impulse at step changes in diameter was used to generate a suitable response. The magnitude of step change in bore diameter was limited by the available machined brick, thus only step changes in bore of $2 \mathrm{~mm}$ were considered. For varying sizes of step changes, it is anticipated that the magnitude of the static component would vary linearly with the magnitude of the step encountered.

Static friction components appear only where there is a step change in bore diameter. It is assumed that if the bore were to transit between two bore sizes gradually over a suitable distance, then no static component would be introduced, though the kinetic friction magnitude would still be dependent upon the bore diameter. Using this assumption, the array of bore diameters is scanned using an algorithm to identify where step changes of bore occur. In this case, a step change of bore is classified as reduction in bore diameter of greater than $1.5 \mathrm{~mm}$ over a traversal of $1 \mathrm{~mm}$. Wherever this condition is met, a standard static response, a triangular pulse is generated for the static component at the corresponding location.

The process for static and kinetic friction is repeated for three brushes and a total frictional response generated for the whole system. Fig.6. shows the individual component responses and the resultant trace.

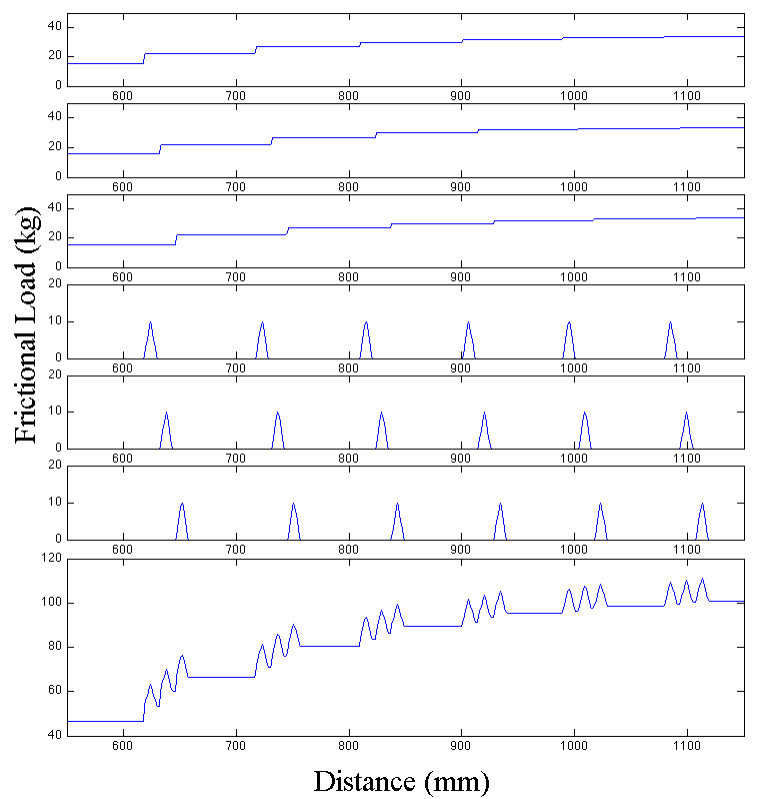

Fig.6. Plot of the individual brush friction kinetic and static components, along with the resultant output. From top to bottom the graphs are Brush 1 Kinetic component, Brush 2 Kinetic Component, Brush 3 Kinetic component, Brush 1 static component, Brush 2 Static component, Brush 3 Static component, Resultant plot. 
Comparison of results

The output from the model is compared to the experimental data and is shown in Fig.7. The model and experimental results show a good correlation. Towards the left of the graph, there appears to be a decrease in friction down to the pure kinetic value that has not been accounted for in the models, but the final value is similar. In addition, the experimental model contains an initial static frictional value that has not been modeled (an assumption within the model is that the fuel assembly is already moving). Note that in the experimental data, this is described as a single spike, which is expected as all three brushes are inside the brick bore to begin with and occur simultaneously, with regards overcoming the initial static friction.

To quantify the correlation between the modeled and measured results, a plot of the residuals is shown in Fig.8. The average residual has a magnitude $-2.68 \mathrm{~kg}$ suggesting the model slightly underestimates the measurements. In addition, there are spikes in the residuals corresponding to the location of the step change in bore suggests that slight improvements to the static friction element of model could be made.

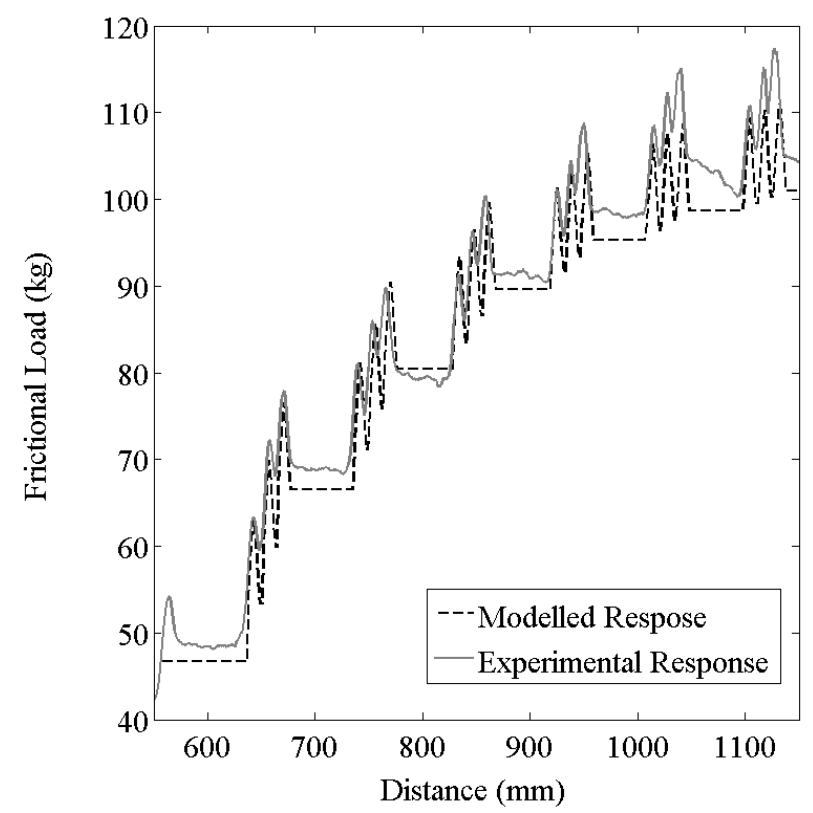

Fig.7. Plot of the modeled frictional response compared to the experimental friction response

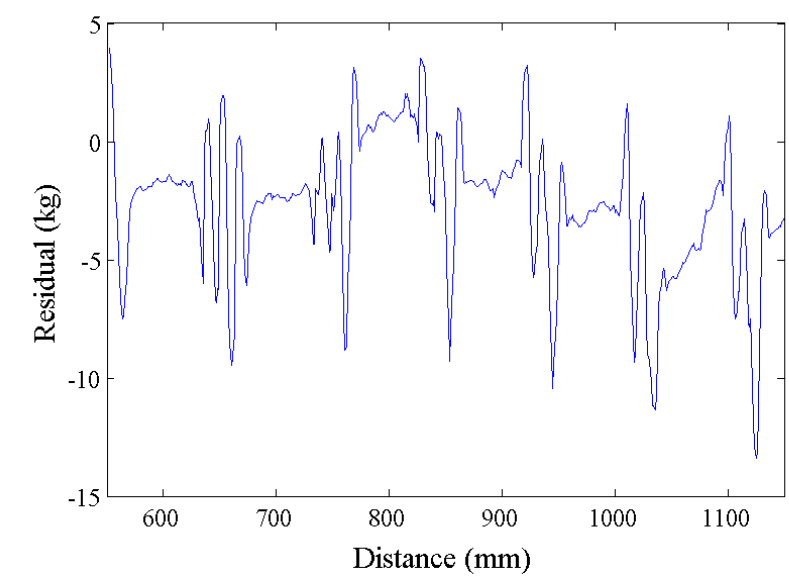

Fig.8. Plot of the residuals between the modeled frictional response compared to the experimental friction response

Upper brush friction

As the lower stabilizing brushes on the fuel assembly pass through the core, the upper stabilizing brush passes through a guide tube fixed to the top of the graphite, into a open region where the hot gas collects and then up through a standpipe into the charge machine. The diameter of the upper stabilizing brush is smaller than the lower brushes and both the guide tube and standpipe generally have sufficiently large diameters that there is no interaction between them and the upper stabilizing brush. However, at several points, particularly the first section of the guide tube and around a piston seal bore, there are regions where the upper 
brush interacts with the guide tube, resulting in a frictional component. The guide tube is manufactured from steel, and not subject to the same level of irradiation as the graphite core. There is, therefore, no appreciable variation in the bore diameter across the guide tube. The entrance and exit to the piston seal bore section is tapered and as a result, the static effects encountered with the lower brushes are not considered to be significant for the upper brush and are therefore not included in the model. The resultant model for the upper stabilizing brush is relatively simple, comprising of two regions where a constant frictional load is applied and zero frictional load in all other regions.

\section{Aerodynamic effects}

In the core of an AGR nuclear power plant, $\mathrm{CO}_{2}$ is used as the coolant. When the reactor is refueled at power the pressure and temperature inside the core is approximately $40 \mathrm{bar}$ and $450^{\circ} \mathrm{C}$ and the flow of $\mathrm{CO}_{2}$ is such that it interacts with the fuel assembly, providing support for some of its weight. This is illustrated in Fig.9. which shows the nose of the assembly situated within the channel and gas flowing up from the bottom of the channel providing an upward force on the fuel assembly.

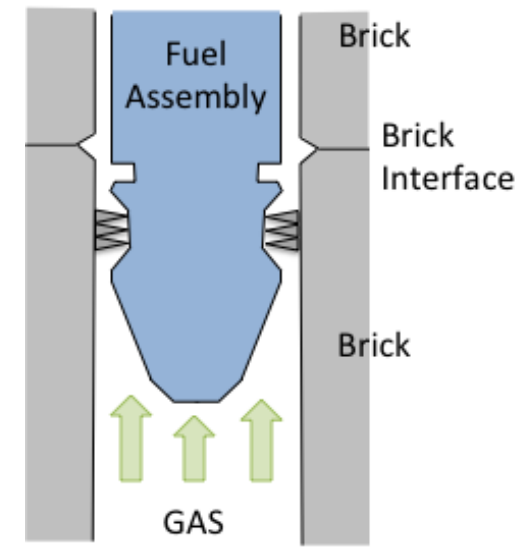

Fig.9. Diagram of fuel assembly sitting in fuel channel illustrating the coolant gas flow

Fuel can also be exchanged during statutory outages, where the reactor is depressurized and the core conditions are very different, in particular the volume flow rate of the coolant. During depressurized refueling, there is no contribution from the aerodynamic effects. Quantifying the gas effects was undertaken using the approach first outlined in Yang et al, 2010, which states if the measured response from the charge and discharge of given fuel channel, at similar reactor conditions, is summed then the frictional components will cancel, leaving just the effects of the aerodynamic component and the absolute weight of the fuel assembly. Fig.10. and Fig.11. provide examples of this from pressurized and depressurized refueling activities respectively. For depressurized refueling the variations in charge and discharge effectively cancel, leaving a linear response, whereas for pressurized events there is a step change in load in the first third of the graph, corresponding to a point where the top of the fuel assembly enters the standpipe and meets gas flowing down from the charge machine. The net result is a downward force that acts on the fuel assembly, increasing the measured load.

Three major assumptions have been made when interpreting and modeling the aerodynamic effects. Firstly, the plot of the aerodynamic effects shown in Fig.11. is not completely linear, but has a series of regularly spaced trough-and-peak features. These correspond to the locations of the brick interfaces and are caused by the chamfered edge of the brick leading to a step change in bore diameter which introduces a static component similar to the ones seen in the experiment involving the lower brush friction. This bore diameter change is shown in Fig.9. Due to the different direction of travel between charge and discharge movements, the static friction components introduced will be offset from each other in the charge and discharge traces. These peaks should effectively be ignored when determining the influence of the aerodynamic components, as it is unlikely that aerodynamic effects of the coolant gas flow would cause such pronounced features over such a short period of time. The second major assumption with this approach is that the weight of the fuel assembly is the same between insertion and removal. This is a reasonable assumption given the same manufacturing procedures and components are used to produce the fuel assemblies and that the weight lost through fission is far smaller than the weight of the fuel assembly itself, which is approximately $2760 \mathrm{~kg}$.

The third major assumption is that new and irradiated brushes will respond in a similar manner, and that dwelling in the core of the reactor for 7-8 years will not significantly affect the properties such as the coefficient of friction of the steel bristles. Gaining these material properties in practice would require experimental work, such as accelerated ageing of brushes in a materials test reactor, followed by a testing to determine the material properties of the irradiated brushes. This is outside the scope of this work, and therefore, the approximation that the response will be similar is made. 
Charge V Discharge Pressurized Refuelling

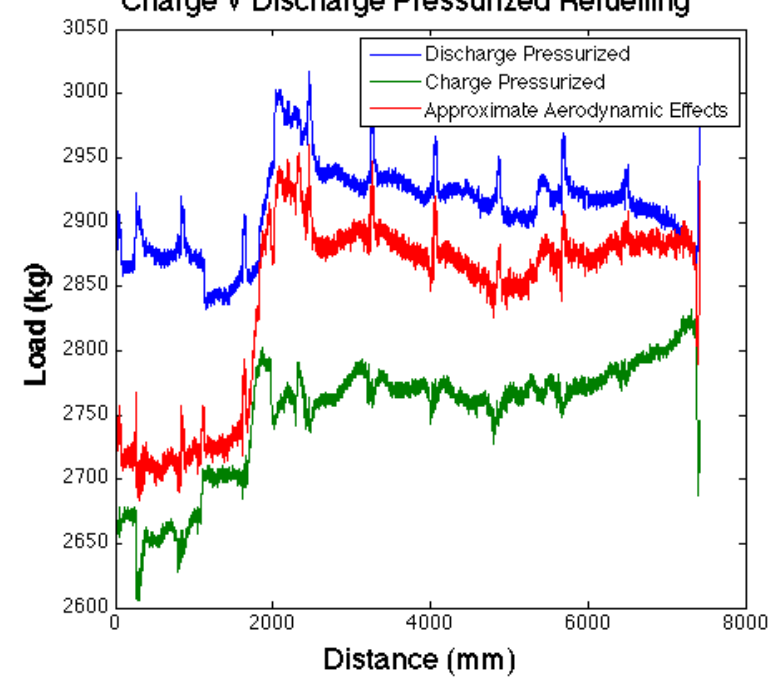

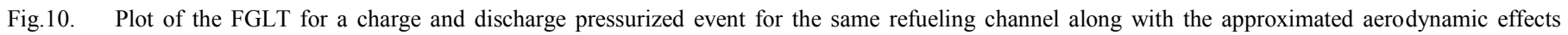
resulting from the summation of both charge and discharge signals (including a $2760 \mathrm{~kg}$ offset, equal to the mass of the fuel assembly).

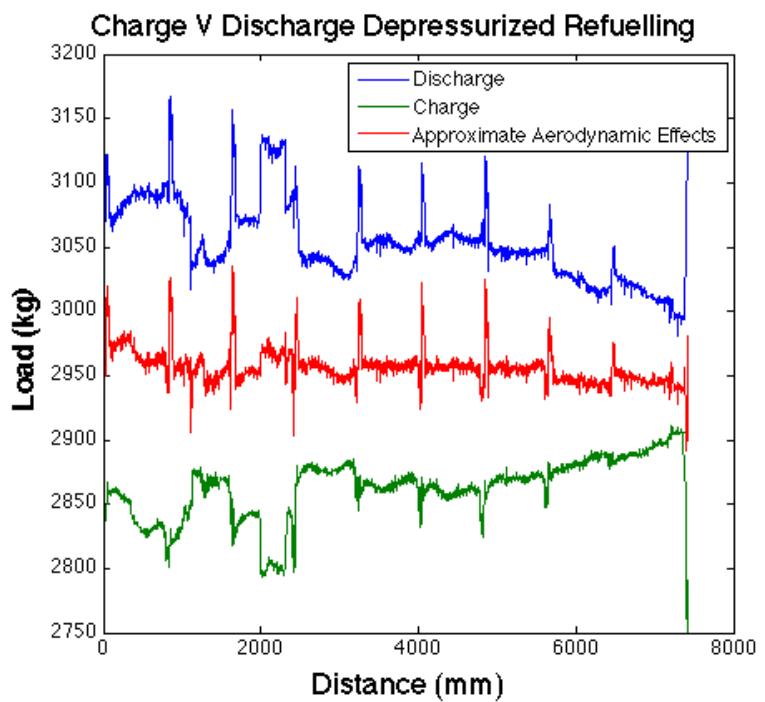

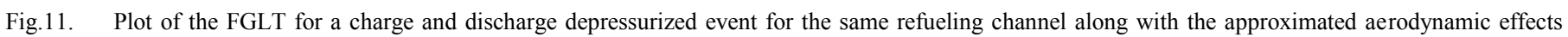
resulting from the summation of both charge and discharge signals (including a $2960 \mathrm{~kg}$ offset, equal to the mass of the fuel assembly).

In Yang et al., 2010 the gas effects were explored for a small number of examples. This was enough to demonstrate the feasibility of the technique, however additional empirical evidence is required to validate the modeling approach. Fig.12. shows the response of the approximate aerodynamic effects from 200 pressurized refueling events taken from four different reactor cores. The data has been significantly down-sampled and smoothed as it is the overall shape of the response that is of most interest. There is some variation in the traces, which is to be expected from slightly different gas flows, however, a broad approximation of the aerodynamic effects can be made from the data.

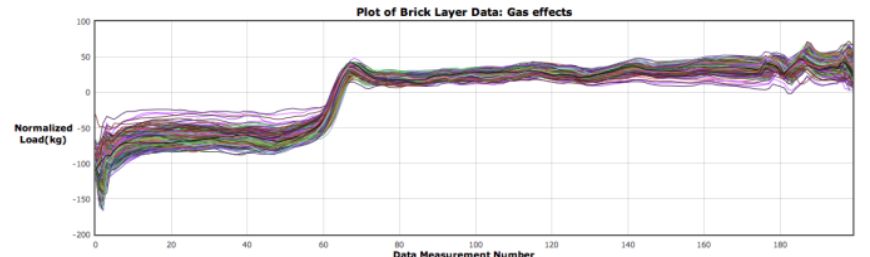

Fig.12. Plot of the approximate aerodynamic effects of 200 pressurized refueling events. Note that the data has been significantly down-sampled and smoothed to facilitate plotting the data. 
The model approximation of the aerodynamic effects for a pressurized refueling event comprises of three sections as follows; a flat region of constant value, a step response with some overshoot and another region of constant value. For depressurized refueling events there is no aerodynamic component.

\section{Combined model}

The individual model components (the lower brush friction, the upper brush friction, the aerodynamic effects and the static weight of the fuel assembly) can be combined to generate an expected fuel grab load trace for bore measurements for a given channel for both pressurized and depressurized reactor refueling conditions. Furthermore, for a given set of bore diameters, both fuel insertion (charge) and fuel removal (discharge) traces can be generated. Fig.13. shows an example of a generated discharge response from the model compared to the actual response for a single pressurized refueling event. The top plot shows the upper stabilizing brush component, the next plot shows the aerodynamic gas effect component, the third plot shows the lower brush friction component and the bottom graph shows the combined model compared with the actual measured values.
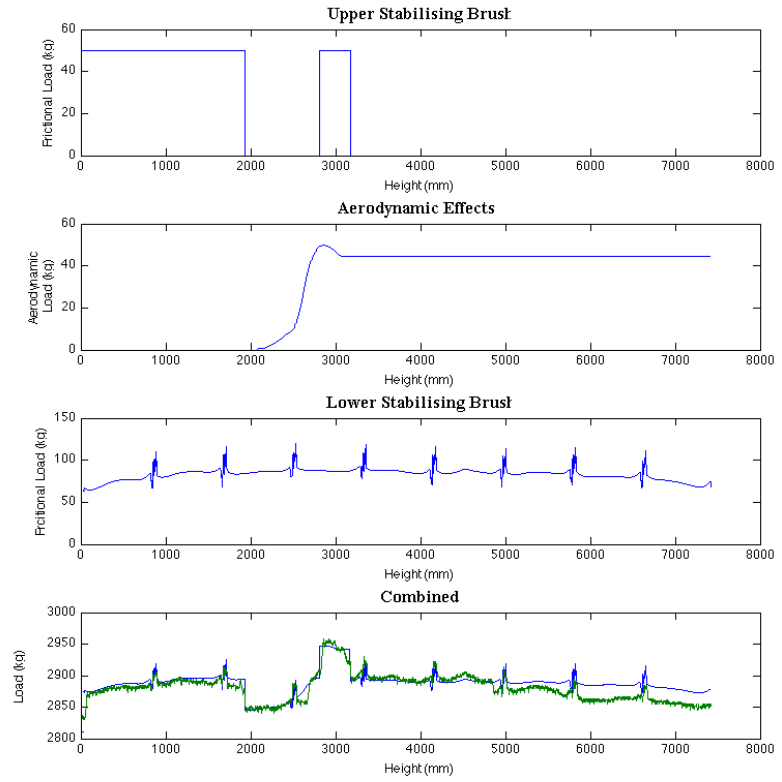

Fig.13. Plot of the modeled response for a pressurized fuel removal compared to the actual measured response taken from a planned outage at an AGR station (shown as the green trace in the bottom diagram). Note that a constant value of $2760 \mathrm{~kg}$ representing the fuel assembly mass was used as the base value, with the individual components added to it to produce the final load shown in the bottom graph. For all graphs, the left hand side equates to the bottom of the reactor core and the right hand side equates to the top of the core region. The guide tube, hotbox and standpipe regions are not shown on these graphs.

Fig.14. shows the results when the bore data is provided in the opposite direction, thus generating the charge response. This time, the frictional components will support some of the weight of the assembly as it is inserted and thus are subtracted from the base weight of the fuel assembly. The aerodynamic gas effect still acts in the same direction and still act as a downward force while the fuel assembly is in the standpipe. 

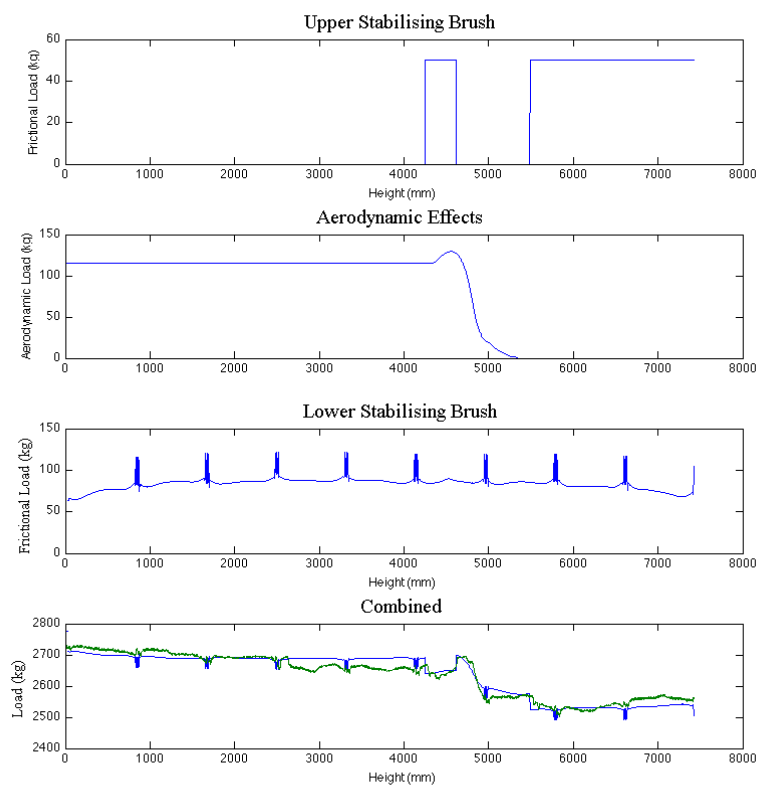

Fig.14. Plot of the modeled response for a pressurized fuel insertion compared to the actual measured response taken from a planned outage at an AGR station (shown as the green trace in the bottom diagram). The same input bore diameter data is used, but run through the model in reverse, hence the upper stabilizing brush and aerodynamic effects are also reversed.

Though this is useful for understanding the FGLT response, from a condition monitoring perspective it is more valuable to generate the resulting bore profile from a measured FGLT trace. Fortunately, the model components are reversible (over the required bore range) and therefore by supplying the measured FGLT as an input, the estimated bore profile can be generated. An example of this is shown in Fig.15. which shows the simulated bore diameter compared with the actual bore diameter measurements taken during a planned outage at an operating AGR station within the UK.

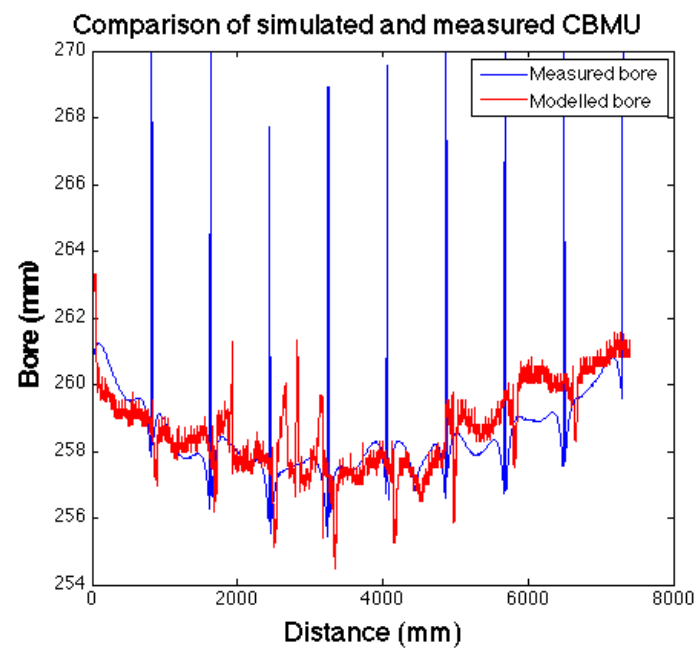

Fig.15. Model output showing the modeled estimation of the bore compared to the actual bore measurements taken from the channel bore monitoring unit (CBMU).

It should be noted that there is an underlying assumption with regards to the presentation of the bore diameter information. The device that makes the in-core inspections takes measurements at multiple different angles, then takes an average of these measurements to get the average bore diameter as in reality the bore is not a perfect circle. Similarly, the impact of the friction will be the average contribution by the brushes around the full circumference of the fuel assembly. This is a recognized limitation of the measurement equipment, but for the purposes of understanding the average bore, and identifying potential anomalies it is sufficient. 


\section{CONCLUSIONS AND FUTURE WORK}

This paper has described an approach for inferring core condition of an AGR through isolating and modeling the various sources which contribute to the final FGLT signal. The issue that the source data was not originally designed as a measure of core condition has been addressed by understanding the underlying process and modeling the individual elements. These models have then been validated through a suitable set of laboratory experiments. This has enhanced the value of the routinely gathered monitoring data by providing additional information relating to the health of the core, and supplementing the existing program of inspection activities undertaken during power plant outages. This addition measure provides increased confidence in the current core health and acts as an additional supporting input into the wider evidence required to continue and extend the operational lifetimes of ageing nuclear power plants within the UK.

\section{ACKNOWLEDGMENT}

This work was funded by EDF Energy. The views presented by the authors do not represent the views of EDF Energy.

\section{REFERENCES}

Brocklehurst, J.E. and Kelly, B.T., 1993. Analysis of the dimensional changes and structural changes in polycrystalline graphite under fast neutron irradiation, Carbon $31(1), 155-178$.

Coble J. B., Ramuhalli, P., Bond, L. J., Hines, J. W., Upadhyaya, B. R., 2012. Prognostics and Health Management in Nuclear Power Plants: A review of Technologies and Applications, PNNL 21515, Pacific National Northwest Lab., Richmond, WA

Cole-Baker, A., and Reed, J., 2007, Measurement of AGR graphite fuel brick shrinkage and channel distortion. In: Neighbour, G.B. (Ed.), Management of Ageing Processes in Graphite Reactor Cores, Special Publication No. 309. RSC Publishing, The Royal Society of Chemistry, Cambridge, UK, 2007, pp. 201208.

Hashemian, H. M., 2010. Applying Online Monitoring for Nuclear Power Plant Instrumentation and Control, IEEE Transactions on Nuclear Science, vol. 57, no. 5, pp. 2872-2878, 2010.

Hashemian, H. M., 2011 “On-line monitoring applications in nuclear power plants,” Progress in Nuclear Energy, vol. 53, no. 2, pp. 167-181

He, S. and Gotts, J.A., 2005. A Computational Study of the Effect of Distortions of the Moderator Cooling Channel of the Advanced Gas-cooled Reactor. Nuclear Engineering and Design No. 235, pp.965-982.

Hines, J. W. and Seibert, R., 2006. Technical review of on-line monitoring techniques for performance assessment, U.S. Nuclear Regulatory Commission, Washington, D.C., NUREG/CR-6895, Vol. 1

Li, H, Marsden, B. J. and Fok, S. L., 2004. Relationship between nuclear graphite moderator brick bore profile measurement and irradiation-induced dimensional change, Nuclear Engineering and Design, Vol 22, pp237-247

Ma, J. and Jiang, J. 2011 “Applications of fault detection and diagnosis methods in nuclear power plants: A review," Progress in Nuclear Energy, vol. 53, no. 3, pp. $255-266$

Marsden, B.J., Hall, G.N., Wouters, O., Vreeling, J.A. and Van Der Laan, J., 2008, Dimensional and material property changes to irradiated Gilsocarbon graphite irradiated between 650 and $750{ }^{\circ} \mathrm{C}$. Journal of Nuclear Materials $381(1-2), 62-67$.

Neighbour, G.B., 2000, Modelling of dimensional changes in irradiated nuclear graphites. Journal of Physics D: Applied Physics 22, $2000,2966-2972$.

Randall, R. B., 2004. State of the art in monitoring rotating machinery-part 1. Sound and Vibration 38.3, pp 14-21.

Shennan, J.V., 1983. “Graphite R\&D Reveals Long Life for AGRs”, ATOM. No. 323, pp 188-191

Simmons, J.H.W. and Perks, A.J., 1965. Dimensional Changes and Radiation Creep in Graphite at Very High Neutron Doses, Nature, Volume 206, Issue 4984, $610-611$

Skelton, J., 2007. Station core monitoring - Experimental work to relate HPB/HNB grab trip load to bore shrinkage, Amec NNC Internal Report, Issue 4, 2007

Steer, A. G., 2005. AGR Core Design, Operation and Safety Functions,” in Managing of Ageing Processes in Graphite Reactor Cores, G. Neighbour, Ed. Royal Society of Chemistry, 2005, pp. 11-18

Stephen, B., West, G.M., Galloway, S. and McArthur, S.D.J., 2009. The Use of Hidden Markov Models for Anomaly Detection in Nuclear Core Condition Monitoring, IEEE Transactions in Nuclear Science, vol. 56, issue 2, pp. 453-461

West, G. M., Jahn, G. J., McArthur, S. D. J., McDonald, J. R. and Reed, J., 2006. Data Mining Reactor Fuel Grab Load Trace Data to Support Nuclear Core Condition Monitoring, IEEE Transactions on Nuclear Science, vol. 53

West, G.M., McArthur, S.D.J. and Towle, D., 2011. Knowledge-Directed Characterization of Nuclear Power Plant Reactor Core Parameters, Journal of Nuclear Engineering and Design, Volume 241 Issue 9, pp 4013-4025.

Yang, E. Grimble, M. J. West, G. M. Inzerillo, S., Katebi M.R. and McArthur, S.D.J., 2010. Model-based estimation and filtering for condition monitoring of AGR nuclear graphite cores, UKACC International Conference on CONTROL 
Dr Graeme West is a research fellow at the Institute for Energy and Environment in the Department of Electronic and Electrical Engineering at the University of Strathclyde. He received a BEng (Hons) degree in Electrical and Mechanical Engineering and a PhD in Electrical Engineering in 1998 and in 2002 respectively, both from the University of Strathclyde.

His current research interests include intelligent system applications and data mining within power engineering and in particular applying the techniques to applications within the nuclear industry.

Chris Wallace is a Research Assistant at the University of Strathclyde researching the application of Multi Agent Systems for data mining and data fusion of condition monitoring data from nuclear power stations. His research interests include the application of AI techniques to condition monitoring and engineering decision support and web technologies for human interface.

Prof. Stephen McArthur (M93, SM07) received his B.Eng. (Hons) and PhD degrees from the University of Strathclyde in 1992 and 1996 respectively. He is a Professor in the Institute for Energy and Environment, within the Department of Electronic and Electrical Engineering. He is Director of the EDF Energy Advanced Diagnostics Centre. He chaired the IEEE PES Working Group on Multi-agent Systems and the IEEE Intelligent Systems subcommittee. His research interests include intelligent system applications in power, condition monitoring, fault diagnosis, smart grids and multi-agent systems. 